\title{
New Probability Distributions in Astrophysics: III. The Truncated Maxwell-Boltzmann Distribution
}

\author{
Lorenzo Zaninetti \\ Physics Department, Turin, Italy \\ Email: zaninetti@ph.unito.it
}

How to cite this paper: Zaninetti, L. (2020) New Probability Distributions in Astrophysics: III. The Truncated Maxwell-Boltzmann Distribution. International Journal of Astronomy and Astrophysics, 10, 191-202. https://doi.org/10.4236/ijaa.2020.103010

Received: June 17, 2020

Accepted: August 16, 2020

Published: August 19, 2020

Copyright $\odot 2020$ by author(s) and Scientific Research Publishing Inc. This work is licensed under the Creative Commons Attribution International License (CC BY 4.0)

http://creativecommons.org/licenses/by/4.0/

\begin{abstract}
The Maxwell-Boltzmann (MB) distribution for velocities in ideal gases is usually defined between zero and infinity. A double truncated MB distribution is here introduced and the probability density function, the distribution function, the average value, the rth moment about the origin, the root-mean-square speed and the variance are evaluated. Two applications are presented: 1) a numerical relationship between root-mean-square speed and temperature, and 2) a modification of the formula for the Jeans escape flux of molecules from an atmosphere.
\end{abstract}

Keywords

05.20.-y Classical Statistical Mechanics, 05.20.Dd Kinetic Theory

\section{Introduction}

The Maxwell-Boltzmann (MB) distribution, see [1] [2], is a powerful tool to explain the kinetic theory of gases. The range in velocity of this distribution spans the interval $[0, \infty]$, which produces several problems:

1) The maximum velocity of a gas cannot be greater than the velocity of light, c.

2) The kinetic theory is developed in a classical environment, which means that the involved velocities should be smaller than $\approx 1 / 10 c$.

These items point toward the hypothesis of an upper bound in velocity for the MB. We will now report some approaches, including an upper bound in velocity: the ion velocities parallel to the magnetic field in a low density surface of a ionized plasma [3]; propagation of longitudinal electron waves in a collisionless, homogeneous, isotropic plasma, whose velocity distribution function is a trun- 
cated MB [4]; fast ion production in laser plasma [5]; the release of a dust particle from a plasma-facing wall [6]; an explanation of an anomaly in the Dark Matter (DAMA) experiment [7]; a distorted MB distribution of epithermal ions observed associated with the collapse of energetic ions [8]; and deviations to $\mathrm{MB}$ distribution that could have observable effects which can be measured trough the vapor spectroscopy at an interface [9]. However, these approaches do not clearly cover the effect of introducing a lower and an upper boundary in the MB distribution, which is the subject that will be analyzed in this paper.

This paper is structured as follows. Section 2 reviews the basic statistics of the $\mathrm{MB}$ distribution and it derives a new approximate expression for the median. Section 3 introduces the double truncated MB and it derives the connected statistics. Section 4 derives the relationship for root-mean-square speed versus temperature in the double truncated MB. Finally, Section 5.2 derives a new formula for Jeans flux in the exosphere.

\section{The Maxwell-Boltzmann Distribution}

Let $V$ be a random variable defined in $[0, \infty]$; the $\mathrm{MB}$ probability density function (PDF), $f(v ; a)$, is

$$
f(v ; a)=\frac{\sqrt{2} v^{2} \mathrm{e}^{-\frac{1}{2} v^{2}} a^{2}}{\sqrt{\pi} a^{3}},
$$

where $a$ is a parameter and $v$ denotes the velocity, see [1] [2]. Conversion to the physics is done by introducing the variable $a$, which is defined as

$$
a=\sqrt{\frac{k T}{m}},
$$

where $m$ is the mass of the gas molecules, $k$ is the Boltzmann constant and $T$ is the thermodynamic temperature. With this change of variable, the MB PDF is

$$
f_{p}(v ; m, k, T)=\frac{\sqrt{2} v^{2} \mathrm{e}^{-\frac{1}{2} v^{2} m}}{\sqrt{\pi}\left(\frac{k T}{m}\right)^{\frac{3}{2}}},
$$

where the index $p$ stands for physics. The distribution function (DF), $F(x ; a)$, is

$$
\begin{gathered}
F(v ; a)=\frac{\sqrt{2} a^{2}\left(a \sqrt{\pi} \sqrt{2} \operatorname{erf}\left(\frac{1}{2} \frac{\sqrt{2} v}{a}\right)-2 v \mathrm{e}^{-\frac{1}{2} \frac{v}{2}^{2}}\right)}{2 \sqrt{\pi} a^{3}} \\
F_{p}(v)=\frac{\left(\left(\frac{k T}{m}\right)^{\frac{3}{2}} \sqrt{\pi} \sqrt{2} \operatorname{erf}\left(\frac{1}{2} \sqrt{2} v \frac{1}{\sqrt{\frac{k T}{m}}}\right) m-2 v \mathrm{e}^{-\frac{1}{2} \frac{v^{2} m}{k T}} k T\right)}{2 \sqrt{\pi}\left(\frac{k T}{m}\right)^{\frac{3}{2}} m} .
\end{gathered}
$$


The average value or mean, $\mu$, is

$$
\begin{gathered}
\mu(a)=2 \frac{\sqrt{2} a}{\sqrt{\pi}}, \\
\mu(m, k, T)_{p}=2 \frac{\sqrt{2}}{\sqrt{\pi}} \sqrt{\frac{k T}{m},}
\end{gathered}
$$

the variance, $\sigma^{2}$, is

$$
\begin{gathered}
\sigma^{2}(a)=\frac{a^{2}(-8+3 \pi)}{\pi} \\
\sigma^{2}(m, k, T)_{p}=\frac{k T(-8+3 \pi)}{m \pi} .
\end{gathered}
$$

The rth moment about the origin for the MB distribution is, $\mu_{r}^{\prime}$, is

$$
\begin{aligned}
\mu_{r}^{\prime}(a) & =\frac{2^{r / 2+1} a^{r} \Gamma\left(r / 2+\frac{3}{2}\right)}{\sqrt{\pi}} \\
\mu_{r}^{\prime}(m, k, T)_{p} & =\frac{2^{r / 2+1}\left(\sqrt{\frac{k T}{m}}\right)^{r} \Gamma\left(r / 2+\frac{3}{2}\right)}{\sqrt{\pi}},
\end{aligned}
$$

where

$$
\Gamma(z)=\int_{0}^{\infty} \mathrm{e}^{-t} t^{z-1} \mathrm{~d} t,
$$

is the gamma function, see [10]. The root-mean-square speed, $v_{r m s}$, can be obtained from this formula by inserting $r=2$

$$
\begin{gathered}
v_{r m s}(a)=\sqrt{3} a \\
v_{r m s}(m, k, T)_{p}=\sqrt{3} \sqrt{\frac{k T}{m}},
\end{gathered}
$$

see Equations (7-10-16) in [11]. This equation allows us to derive the temperature once the root-mean-square speed is measured

$$
T=\frac{1 v_{r m s}^{2} m}{3} .
$$

The coefficient of variation $(C V)$ is

$$
C V=\frac{\sigma(a)}{\mu(a)}=\sqrt{\frac{3}{8} \pi-1},
$$

which is constant. The first three rth moments about the mean for the MB distribution, $\mu_{r}(a)$, are

$$
\begin{array}{r}
\mu_{2}(a)=\frac{a^{2}(-8+3 \pi)}{\pi} \\
\mu_{3}(a)=-2 \frac{a^{3} \sqrt{2}(5 \pi-16)}{\pi^{3 / 2}} \\
\mu_{4}(a)=\frac{a^{4}\left(15 \pi^{2}+16 \pi-192\right)}{\pi^{2}} .
\end{array}
$$


The mode is at

$$
\begin{gathered}
v(a)=\sqrt{2} a \\
v(m, k, T)_{p}=\sqrt{2} \sqrt{\frac{k T}{m} .}
\end{gathered}
$$

An approximate expression for the median can be obtained by a Taylor series of the DF around the mode. The approximation formula is

$$
\begin{gathered}
v(a)=-\frac{1}{4} a\left(-6+\mathrm{e}\left(\operatorname{erf}(1)-\frac{1}{2}\right) \sqrt{\pi}\right) \sqrt{2}, \\
v(m, k, T)_{p}=-\frac{1}{4} \sqrt{\frac{k T}{m}}\left(-6+\mathrm{e}\left(\operatorname{erf}(1)-\frac{1}{2}\right) \sqrt{\pi}\right) \sqrt{2},
\end{gathered}
$$

which has a percent error, $\delta$, of $\delta \approx 0.04 \%$ in respect to the numerical value. The entropy is

$$
\begin{gathered}
\ln (\sqrt{2} \sqrt{\pi} a)-\frac{1}{2}+\gamma, \\
\ln \left(\sqrt{2} \sqrt{\pi} \sqrt{\frac{k T}{m}}\right)-\frac{1}{2}+\gamma,
\end{gathered}
$$

where $\gamma$ is the Euler-Mascheroni constant, which is defined as

$$
\gamma=\lim _{n \rightarrow \infty}\left(1+\frac{1}{2}+\frac{1}{3}+\cdots+\frac{1}{n}-\ln n\right)=0.57721 \cdots
$$

see [10] for more details. The coefficient of skewness is

$$
\frac{(-10 \pi+32) \sqrt{2}}{(-8+3 \pi)^{\frac{3}{2}}} \approx 0.48569,
$$

and the coefficient of kurtosis is

$$
\frac{15 \pi^{2}+16 \pi-192}{(-8+3 \pi)^{2}} \approx 3.10816 .
$$

According to [12], a random number generation can be obtained via inverse transform sampling when the distribution function or cumulative distribution function, $F(x)$, is known: 1 ) a pseudo number generator gives a random number $R$ between zero and one; 2) the inverse function $x=F^{-1}(R)$ is evaluated; and 3) the procedure is repeated for different values of $R$. In our case, the inverse function should be evaluated in a numerical way by solving for $v$ the following nonlinear equation

$$
\begin{gathered}
F(v ; a)-R=0, \\
F(v ; m, k, T)_{p}-R=0,
\end{gathered}
$$

where $F(v)$ and $F_{p}(v)$ are the two DF represented by Equations (4) and (5). As a practical example, by inserting in Equation (29) $a=1$ and $R=0.5$, we obtain in a numerical way $v=1.538$. 


\section{The Double Truncated Maxwell-Boltzmann Distribution}

Let $V$ be a random variable that is defined in $\left[v_{l}, v_{u}\right]$; the double truncated version of the Maxwell-Boltzmann PDF, $f_{t}\left(v ; a, v_{l}, v_{u}\right)$, is

$$
f_{t}\left(v ; a, v_{l}, v_{u}\right)=v^{2} \mathrm{e}^{-\frac{1 v^{2}}{2} a^{2}}
$$

where

$$
C=\frac{-2}{C D}
$$

where

$$
\begin{aligned}
& C D=a^{2}\left(-a \sqrt{\pi} \sqrt{2} \operatorname{erf}\left(\frac{1}{2} \frac{\sqrt{2} v_{u}}{a}\right)+a \sqrt{\pi} \sqrt{2} \operatorname{erf}\left(\frac{1}{2} \frac{\sqrt{2} v_{l}}{a}\right)\right. \\
& \left.+2 v_{u} \mathrm{e}^{-\frac{1}{2} \frac{v_{u}^{2}}{a^{2}}}-2 v_{l} \mathrm{e}^{-\frac{1}{2} v_{l}^{2}} a^{2}\right)
\end{aligned}
$$

and $\operatorname{erf}(x)$ is the error function, which is defined as

$$
\operatorname{erf}(x)=\frac{2}{\sqrt{\pi}} \int_{0}^{x} \mathrm{e}^{-t^{2}} \mathrm{~d} t,
$$

see [10]. The physical meaning of $a$ is still represented by Equation (2); however, due to the tendency to obtain complicated expressions, we will omit the double notation. The DF, $F_{t}\left(v ; a, v_{l}, v_{u}\right)$, is

$$
F_{t}\left(v ; a, v_{l}, v_{u}\right)=\frac{C a^{2}\left(\sqrt{\pi} \sqrt{2} a \operatorname{erf}\left(\frac{1}{2} \frac{\sqrt{2} v}{a}\right)-2 v \mathrm{e}^{-\frac{1}{2} v^{2}}\right)}{2} .
$$

The average value $\mu_{t}\left(a, v_{l}, v_{u}\right)$, is

$$
\mu_{t}\left(a, v_{l}, v_{u}\right)=C a^{2}\left(2 \mathrm{e}^{-\frac{1}{2} \frac{v_{l}^{2}}{a^{2}}} a^{2}-2 \mathrm{e}^{-\frac{1}{2} \frac{v_{u}^{2}}{a^{2}}} a^{2}+\mathrm{e}^{-\frac{1}{2} \frac{v_{l}^{2}}{a^{2}}} v_{l}^{2}-\mathrm{e}^{-\frac{1}{2} \frac{v_{u}^{2}}{a^{2}}} v_{u}^{2}\right) .
$$

The rth moment about the origin for the double truncated MB distribution is, $\mu_{r, t}^{\prime}\left(a, v_{l}, v_{u}\right)$,

$$
\mu_{r, t}^{\prime}\left(a, v_{l}, v_{u}\right)=\frac{M N}{r+3}
$$

where

$$
\begin{aligned}
M N= & C 2^{\frac{r}{4}+\frac{5}{4}} a^{2} \times\left(\left(\frac{v_{u}^{2}}{a^{2}}\right)^{-\frac{r}{4}-\frac{1}{4}} v_{u}^{r+1} \mathrm{e}^{-\frac{1}{4} v_{u}^{2}} M_{\frac{r}{4}+\frac{1}{4}, \frac{r}{4}+\frac{3}{4}}\left(\frac{1}{2} \frac{v_{u}^{2}}{a^{2}}\right)\right. \\
& \left.-v_{l}^{r+1} \mathrm{e}^{-\frac{1}{4} \frac{v_{l}^{2}}{a^{2}}} M_{\frac{r}{4}+\frac{1}{4}, \frac{r}{4}+\frac{3}{4}}\left(\frac{1}{2} \frac{v_{l}^{2}}{a^{2}}\right)\left(\frac{v_{l}^{2}}{a^{2}}\right)^{-\frac{r}{4}-\frac{1}{4}}\right)
\end{aligned}
$$

where $M_{\mu, v}(z)$ is the Whittaker $M$ function, see [10]. The root-mean-square speed, $v_{r m s, t}\left(a, v_{l}, v_{u}\right)$, can be obtained from this formula by inserting $r=2$, and is 


$$
v_{r m s, t}\left(a, v_{l}, v_{u}\right)=\sqrt{\frac{N V}{5\left(\frac{v_{u}^{2}}{a^{2}}\right)^{3 / 4}\left(\frac{v_{l}^{2}}{a^{2}}\right)^{3 / 4}}}
$$

where

$$
\begin{aligned}
N V= & 2 C 2^{3 / 4} a^{2}\left(v_{u}^{3} \mathrm{e}^{-1 / 4 \frac{v_{u}^{2}}{a^{2}}} M_{3 / 4,5 / 4}\left(1 / 2 \frac{v_{u}^{2}}{a^{2}}\right)\left(\frac{v_{l}^{2}}{a^{2}}\right)^{3 / 4}\right. \\
& \left.-v_{l}^{3} \mathrm{e}^{-1 / 4 \frac{v_{l}^{2}}{a^{2}}} M_{3 / 4,5 / 4}\left(1 / 2 \frac{v_{l}^{2}}{a^{2}}\right)\left(\frac{v_{u}^{2}}{a^{2}}\right)^{3 / 4}\right) .
\end{aligned}
$$

The variance $\sigma_{t}^{2}\left(a, v_{l}, v_{u}\right)$ is defined as

$$
\sigma_{t}^{2}\left(a, v_{l}, v_{u}\right)=\mu_{2, t}^{\prime}\left(a, v_{l}, v_{u}\right)-\left(\mu_{1, t}^{\prime}\left(a, v_{l}, v_{u}\right)\right)^{2}
$$

and has the following explicit form

$$
\begin{aligned}
& \sigma_{t}^{2}\left(a, v_{l}, v_{u}\right) \\
& =4\left(\left(\left(v_{l}+2 v_{u}\right) a^{2}+v_{l} v_{u}\left(v_{l}+\frac{1}{2} v_{u}\right)\right)\left(a^{2}+1 / 2 v_{u}^{2}\right) C^{2} a^{4} \mathrm{e}^{-\frac{1}{2} \frac{v_{l}^{2}+2 v_{u}^{2}}{a^{2}}}\right. \\
& -2\left(\left(v_{l}+\frac{1}{2} v_{u}\right) a^{2}+\frac{1}{4} v_{l} v_{u}\left(v_{l}+2 v_{u}\right)\right) C^{2} a^{4}\left(a^{2}+\frac{1}{2} v_{l}^{2}\right) \mathrm{e}^{-\frac{1}{2} \frac{2 v_{l}^{2}+v_{u}^{2}}{a^{2}}} \\
& +\left(a^{2}+\frac{1}{2} v_{u}^{2}\right)\left(\operatorname{Cerf}\left(\frac{1}{2} \frac{\sqrt{2} v_{l}}{a}\right) a^{3} \sqrt{2} \sqrt{\pi}\right. \\
& \left.-C \operatorname{erf}\left(\frac{1}{2} \frac{\sqrt{2} v_{u}}{a}\right) a^{3} \sqrt{2} \sqrt{\pi}+4\right) C a^{2}\left(a^{2}+\frac{1}{2} v_{l}^{2}\right) \mathrm{e}^{-\frac{1}{2} v_{l}^{2}+v_{u}^{2}}
\end{aligned}
$$

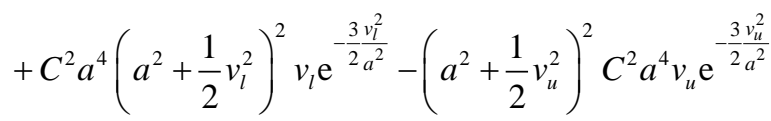

$$
\begin{aligned}
& +\left(\frac{3}{4} a^{2} v_{l}+\frac{1}{4} v_{l}^{3}\right) \mathrm{e}^{-\frac{1}{2} v_{l}^{2}} a^{2}+\left(-\frac{3}{4} a^{2} v_{u}-\frac{1}{4} v_{u}^{3}\right) \mathrm{e}^{-\frac{1}{2} v_{u}^{2}} \\
& -\frac{1}{2}\left(\left(\operatorname{Cerf}\left(\frac{1}{2} \frac{\sqrt{2} v_{l}}{a}\right) a^{3} \sqrt{2} \sqrt{\pi}\right.\right. \\
& \left.-C \operatorname{erf}\left(\frac{1}{2} \frac{\sqrt{2} v_{u}}{a}\right) a^{3} \sqrt{2} \sqrt{\pi}+4\right) C\left(a^{2}+\frac{1}{2} v_{l}^{2}\right)^{2} \mathrm{e}^{-\frac{v_{l}^{2}}{a^{2}}} \\
& +\left(a^{2}+\frac{1}{2} v_{u}^{2}\right)^{2}\left(\operatorname{Cerf}\left(\frac{1}{2} \frac{\sqrt{2} v_{l}}{a}\right) a^{3} \sqrt{2} \sqrt{\pi}\right. \\
& \left.-C \operatorname{erf}\left(\frac{1}{2} \frac{\sqrt{2} v_{u}}{a}\right) a^{3} \sqrt{2} \sqrt{\pi}+4\right) C \mathrm{e}^{-\frac{v_{u}^{2}}{a^{2}}} \\
& \left.\left.+\frac{3}{4} \sqrt{\pi} \sqrt{2}\left(-\operatorname{erf}\left(\frac{1}{2} \frac{\sqrt{2} v_{u}}{a}\right)+\operatorname{erf}\left(\frac{1}{2} \frac{\sqrt{2} v_{l}}{a}\right)\right) a\right) a^{2}\right) C a^{2} .
\end{aligned}
$$


Although the coefficients of skewness and kurtosis for the truncated MB exist, they have a complicated expression.

\section{A Laboratory Application}

The temperature as a function of root-mean-square speed for the $\mathrm{MB}$ is given by Equation (15). In the truncated MB distribution, the temperature can be found by solving the following nonlinear equation

$$
v_{r m s, t}\left(k, m, T, v_{l}, v_{u}\right)=v_{r m s, m},
$$

where $v_{r m s, m}$ is not a theoretical variable but is the root-mean-square speed measured in the laboratory and $v_{r m s, t}$ is given by Equation (39). The laboratory measures of $v_{r m s, m}$ started with [13], where a $v_{r m s, m}=388 \mathrm{~m} / \mathrm{s}$ at $400^{\circ} \mathrm{C}$ was found for a metallic vapor. In the truncated $\mathrm{MB}$ distribution, there are three parameters that can be measured in the laboratory from a kinematical point of view, as follows: the lowest velocity, $v_{l}$; the highest velocity, $v_{u}$; and the root-mean-square speed, $v_{r m s, m}$. Setting for simplicity $v_{l}=0$, we will now explore the effect of the variation of $v_{u}$ on the root-mean-square speed; see Figure 1 . The first example of the influence of the upper limit in velocity on the temperature is given by potassium gas [14] [15], in which molecular mass is $6.492429890 \times 10^{-26} \mathrm{~kg}$. In Figure 2, we evaluate in a numerical way the temperature when $v_{l}=0$ and $v_{u}$ is variable in the case of a measured value of $v_{r m s, m}$.

The second example is given by diatomic nitrogen, $\mathrm{N}_{2}$, in which molecular mass is $4.651737684 \times 10^{-26} \mathrm{~kg}$. In Figure 3, we evaluate the temperature when $v_{l}=0$ and $v_{u}$ is a variable in the case of a measured value of $v_{r m s, m}$.

\section{The Jeans Escape}

The standard formula for the escape of molecules from the exosphere is reviewed in the framework of the MB distribution. A new formula for the Jeans escape is derived in the framework of the truncated MB.

\subsection{The Standard Case}

In the exosphere, a molecule of mass $m$ and velocity $v_{e}$ is free to escape when

$$
\frac{1}{2} m v_{e}^{2}-G \frac{M m}{R_{e x}}=0,
$$

where $G$ is the Newtonian gravitational constant, $M$ is the mass of the Earth, $R_{e x}=R+H$ is the radius of the exosphere, $R$ is the radius of the Earth and $H$ is the altitude of the exosphere. The flux of the molecules that are living in the exosphere $\Phi_{j}$ is

$$
\Phi_{j}=\frac{1}{4} N_{e x} \mu_{e},
$$

where $N_{e x}$ is the number of molecules per unit volume and $\mu_{e}$ is the average velocity of escape. In the presence of a given number of molecules per unit volume, the standard MB distribution in velocities in a unit volume, $f_{m}$, is 


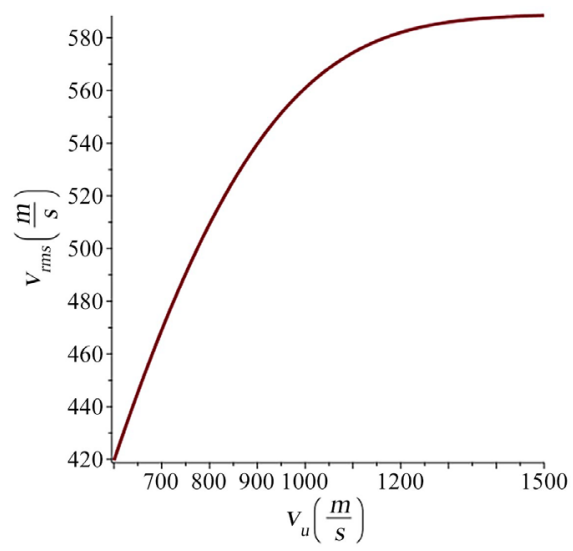

Figure 1. The theoretical root-mean-square speed as a function of the upper limit in velocity (continuous line) and standard value of the temperature (dotted line) when $a=340$ and $v_{l}=0$.

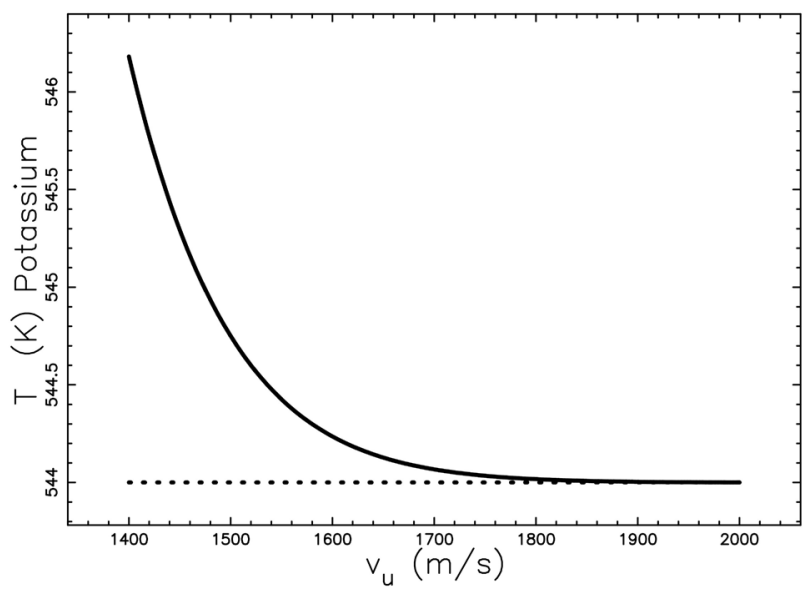

Figure 2. Temperature as a function of the upper limit in velocity for Potassium (continuous line) and standard value of the temperature (dotted line) when $v_{l}=0$ and $v_{r m s, m}=589.111511 \mathrm{~m} / \mathrm{s}$.

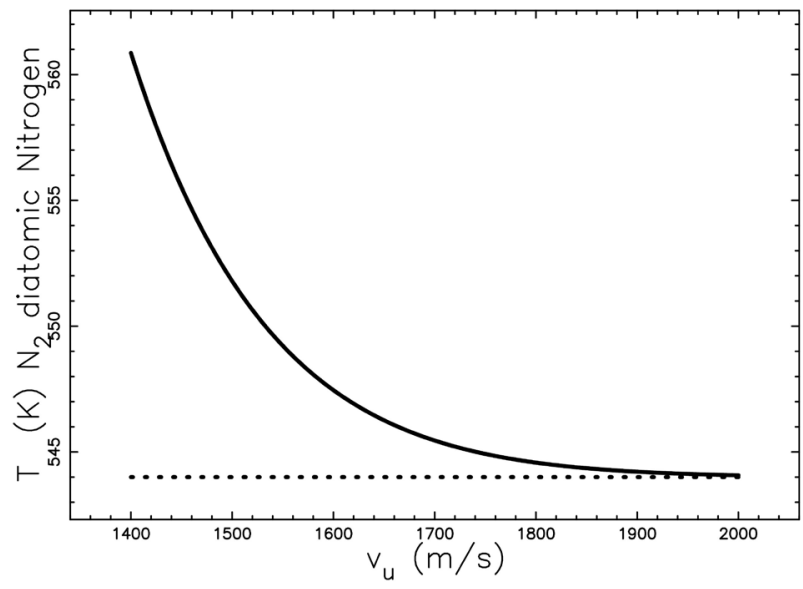

Figure 3. Temperature as a function of the upper limit in velocity for diatomic nitrogen, $\mathrm{N}_{2}$, (continuous line) and standard value of the temperature (dotted line) when $v_{l}=0$ and $v_{r m s, m}=695.9756308 \mathrm{~m} / \mathrm{s}$. 


$$
f_{m}\left(v ; m, k, T, N_{e x}\right)=N_{e x} \frac{\sqrt{2} v^{2} \mathrm{e}^{-\frac{1}{2} \frac{v^{2} m}{k T}}}{\sqrt{\pi}\left(\frac{k T}{m}\right)^{\frac{3}{2}}} .
$$

The average value of escape is defined as

$$
\mu_{e}=\frac{\int_{v_{e}}^{\infty} v f_{m}\left(v ; m, k, T, N_{e x}\right) \mathrm{d} v}{\int_{0}^{\infty} f_{m}\left(v ; m, k, T, N_{e x}\right) \mathrm{d} v} .
$$

In this integral, the following changes are made to the variables

$$
\lambda=\frac{1}{2} \frac{m v^{2}}{k T} \text {. }
$$

Therefore,

$$
\mu_{e}=2\left(\lambda_{e}+1\right) \mathrm{e}^{-\lambda_{e}} \sqrt{2} \sqrt{\frac{k T}{\pi m}},
$$

with

$$
\lambda_{e}=2 \frac{G M}{R_{e x} v_{0}^{2}},
$$

where $v_{0}$ is the mode as represented by Equation (21). The flux is now

$$
\Phi_{j}=\frac{N_{e x}\left(\lambda_{e}+1\right) \mathrm{e}^{-\lambda_{e}} V_{0}}{2 \sqrt{\pi}} .
$$

For more details see [16] [17] [18] [19]. On adopting the parameters of Table 1 the Jeans escape flux for hydrogen is

$$
\Phi_{j}=3.98 \times 10^{11} \text { molecules } \cdot \mathrm{m}^{-2} \cdot \mathrm{s}^{-1},
$$

and

$$
\lambda_{e}=7.78
$$

The Jeans escape flux for Earth at $T=900 \mathrm{~K}$ varies between $\Phi_{j} \approx 2.7 \times 10^{11}$ molecules $\cdot \mathrm{m}^{-2} \cdot \mathrm{s}^{-1}$; see [20] or Figure 1 in [21]. and $\Phi_{j} \approx 4 \times 10^{11}$ molecules $\cdot \mathrm{m}^{-2} \cdot \mathrm{s}^{-1}$, see [22]. Therefore, our choice of parameters is compatible with the suggested interval in flux.

\subsection{The Truncated Case}

The average value of escape for a truncated MB distribution, $\mu_{e, t}$, is

$$
\mu_{e, t}=\frac{\int_{v_{e}}^{\infty} v f_{t}\left(v ; m, k, T, N_{e x}, v_{l}, v_{u}\right) \mathrm{d} v}{\int_{0}^{\infty} f_{m}\left(v ; m, k, T, N_{e x}, v_{l}, v_{u}\right) \mathrm{d} v} .
$$

This integral can be solved by introducing the change of variable as given by Equation (48)

$$
\mu_{e, t}=-2 \frac{\left(\left(\lambda_{u}+1\right) \mathrm{e}^{-\lambda_{u}}-\mathrm{e}^{-\lambda_{e}}\left(\lambda_{e}+1\right)\right) \sqrt{2}}{2 \sqrt{\lambda_{l}} \mathrm{e}^{-\lambda_{l}}-2 \sqrt{\lambda_{u}} \mathrm{e}^{-\lambda_{u}}-\sqrt{\pi} \operatorname{erf}\left(\sqrt{\lambda_{l}}\right)+\sqrt{\pi} \operatorname{erf}\left(\sqrt{\lambda_{u}}\right)} \sqrt{\frac{k T}{m}},
$$

where $\lambda_{1}$ is the lower value of $\lambda$ and $\lambda_{u}$ is the upper value of $\lambda$. The flux 
of the molecules that are living the exosphere in the truncated $\mathrm{MB}, \Phi_{j, t}$, is

$$
\Phi_{j, t}=\frac{N_{e x}\left(\left(\lambda_{u}+1\right) \mathrm{e}^{-\lambda_{u}}-\mathrm{e}^{-\lambda_{e}}\left(\lambda_{e}+1\right)\right) \sqrt{2}}{4 \sqrt{\lambda_{u}} \mathrm{e}^{-\lambda_{u}}+2 \sqrt{\pi} \operatorname{erf}\left(\sqrt{\lambda_{l}}\right)-2 \sqrt{\pi} \operatorname{erf}\left(\sqrt{\lambda_{u}}\right)-4 \sqrt{\lambda_{l}} \mathrm{e}^{-\lambda_{l}}} \sqrt{\frac{k T}{m}} .
$$

The increasing flux of molecules is outlined when one parameter, $\lambda_{1}$, is variable; see Figure 4. In other words, an increase in $\lambda_{1}$ produces an increase in the flux of the molecules. The dependence of the flux when two parameters are variable, $\lambda_{l}$ and $\lambda_{u}$, is reported in Figure 5.

Table 1. Adopted physical parameters for the exosphere.

\begin{tabular}{cc}
\hline Parameter & Value \\
\hline$R_{e x}$ & $6900 \mathrm{~km}$ \\
$T$ & $900 \mathrm{~K}$ \\
$N_{e x}$ & $10^{11} \mathrm{~m}^{-3}$ \\
\hline
\end{tabular}

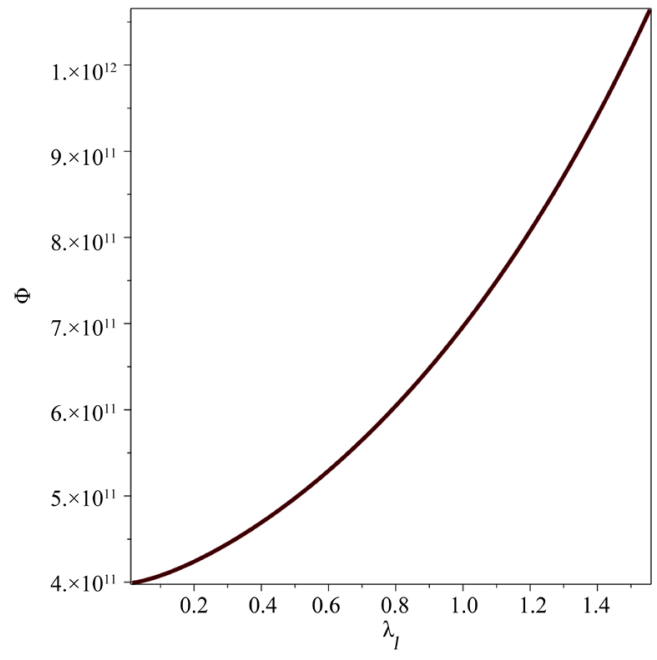

Figure 4. The flux of molecules as a function of $\lambda_{1}$ with parameters as in Table 1, $\lambda_{e}=7.78$ and $\lambda_{u}=1000 \lambda_{e}$.

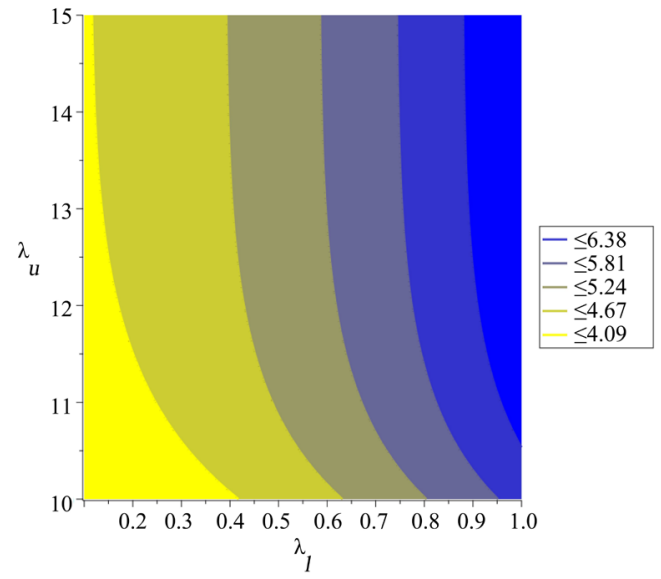

Figure 5. The flux of molecules as a function of $\lambda_{l}$ and $\lambda_{u}$ with parameters as in Table 1. 
These Jeans escape fluxes for Earth are compatible with the observed values that were reported in Section 5.1.

\section{Conclusion}

This paper derived analytical formulae for the following quantities for a double truncated MB distribution: the PDF, the DF, the average value, the rth moment about the origin, the root-mean-square speed and the variance. The traditional correspondence between root-mean-square speed and temperature is replaced by the nonlinear Equation (43). The new formula (56) for the Jeans escape flux of molecules from an atmosphere is now a function of the lower and upper boundary in velocity.

\section{Conflicts of Interest}

The author declares no conflicts of interest regarding the publication of this paper.

\section{References}

[1] Maxwell, J.C. (1860) V. Illustrations of the Dynamical Theory of Gases-Part I. On the Motions and Collisions of Perfectly Elastic Spheres. The London, Edinburgh, and Dublin Philosophical Magazine and Journal of Science, 19, 19-32. https://doi.org/10.1080/14786446008642818

[2] Boltzmann, L. (1872) Weitere Studien über das Wärmegleichgewicht unter Gasmolekülen. Sitzungsberichte Akademie der Wissenschaften, 66, 275-370.

[3] Buzzi, J.M., Doucet, H.J. and Gresillon, D. (1970) Ion Distribution Functions in Collisionless Surface Ionized Plasmas. The Physics of Fluids, 13, 3041. https://doi.org/10.1063/1.1692899

[4] Treguier, J. and Henry, D. (1976) Propagation of Electronic Longitudinal Modes in a Truncated Maxwellian Plasma. Journal of Plasma Physics, 15, 447-461. https://doi.org/10.1017/S0022377800019954

[5] Kishimoto, Y., Mima, K., Watanabe, T. and Nishikawa, K. (1983) Analysis of Fast-Ion velocity Distributions in Laser Plasmas with a Truncated Maxwellian Velocity Distribution of Hot Electrons. The Physics of Fluids, 26, 2308. https://doi.org/10.1063/1.864389

[6] Tomita, Y., Smirnov, R., Nakamura, H., Zhu, S., Takizuka, T. and Tskhakaya, D. (2007) Effect of Truncation of Electron Velocity Distribution on Release of Dust Particle from Plasma-Facing Wall. Journal of Nuclear Materials, 363, 264-269. https://doi.org/10.1016/j.jnucmat.2007.01.025

[7] Fowlie, A. (2017) Halo-Independence with Quantified Maximum Entropy at DAMA/LIBRA. Journal of Cosmology and Astroparticle Physics, 2017, 2-4. https://doi.org/10.1088/1475-7516/2017/10/002

[8] Ida, K., Kobayashi, T., Yoshinuma, M., Akiyama, T., Tokuzawa, T., Tsuchiya, H., Itoh, K. and LHD Experiment Group (2017) Observation of Distorted Maxwell-Boltzmann Distribution of Epithermal Ions in LHD. Physics of Plasmas, 24, Article ID: 122502. https://doi.org/10.1063/1.4999644

[9] Todorov, P., De Aquino Carvalho, J.C., Maurin, I., Laliotis, A. and Bloch, D. (2019) Search for Deviations from the Ideal Maxwell-Boltzmann Distribution for a Gas at 
an Interface. Proceedings of the SPIE, 11047, Article ID: 110470.

[10] Olver, F.W.J., Lozier, D.W., Boisvert, R.F. and Clark, C.W. (2010) NIST Handbook of Mathematical Functions. Cambridge University Press, Cambridge.

[11] Reif, F. (2009) Fundamentals of Statistical and Thermal Physics. Waveland Press, Long Grove, Illinois.

[12] Devroye, L. (1986) General Principles in Random Variate Generation. Springer, New York. https://doi.org/10.1007/978-1-4613-8643-8

[13] Eldridge, J. (1927) Experimental Test of Maxwell's Distribution Law. Physical Review, 30, 931-935. https://doi.org/10.1103/PhysRev.30.931

[14] Miller, R.C. and Kusch, P. (1955) Velocity Distributions in Potassium and Thallium Atomic Beams. Physical Review, 99, 1314-1321. https://doi.org/10.1103/PhysRev.99.1314

[15] Hernandez, H. (2017) Standard Maxwell-Boltzmann Distribution: Definition and Properties. ForsChem Research Reports, Medellin.

[16] Jeans, J.H. (1955) The Dynamical Theory of Gases. Dover, New York.

[17] Shu, F.H. (1982) The Physical Universe. University Science Books, Mill Valley.

[18] Catling, D.C. and Kasting, J.F. (2017) Atmospheric Evolution on Inhabited and Lifeless Worlds. Cambridge University Press, Cambridge, 129.

https://doi.org/10.1017/9781139020558

[19] Owen, J.E. (2019) Atmospheric Escape and the Evolution of Close-in Exoplanets. Annual Review of Earth and Planetary Sciences, 47, 67-90. https://doi.org/10.1146/annurev-earth-053018-060246

[20] Vidal-Madjar, A., Blamont, J.E. and Phissamay, B. (1974) Evolution with Solar Activity of the Atomic Hydrogen Density at 100 Kilometers of Altitude. Journal of Geophysical Research, 79, 233-241. https://doi.org/10.1029/JA079i001p00233

[21] Liu, W., Chiao, M., Collier, M.R., et al. (2017) The Structure of the Local Hot Bubble. The Astrophysical Journal, 834, 33-38. https://doi.org/10.3847/1538-4357/834/1/33

[22] Bertaux, J. (1974) Lhydrogène atomique dans lexosphère terrestre: Mesures dintensité et de largeur de raie de lémission lyman alpha à bord du satellite ogo 5 et interprétation. These détat, Université Paris. 